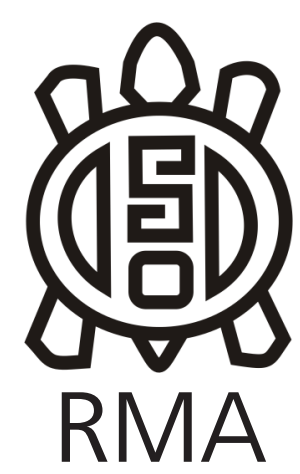

Dossier

\title{
Trayectorias cruzadas de gente y materias primas: Movilidad entre Puna y Valles en el Noroeste de Argentina durante el Holoceno Medio
}

\author{
Intersected Paths of People and Raw Materials: Mobility \\ between Puna and Valleys of the Northwest of Argentina \\ during the Middle Holocene
}

Jorge Gabriel Martínez* y Jorge Alejandro Funes Coronel**

*Instituto Superior de Estudios Sociales-CONICET, Instituto de Arqueología y Museo-UNT.

E-mail: jormartin1969@yahoo.com.ar

**Instituto de Arqueología y Museo-UNT. E-mail:chorchfunes@gmail.com

\begin{abstract}
Resumen
Se aborda en este trabajo el estudio comparativo de evidencias arqueológicas pertenecientes a dos áreas geográficas diferenciadas dentro del noroeste de Argentina: Antofagasta de la Sierra en la Puna meridional (Catamarca) y Quebrada de Los Corrales situada en un sector de Valles intermontanos en el noroeste de Tucumán. Ambas áreas cuentan con secuencias ocupacionales de larga duración dentro del Holoceno, donde hemos podido identificar la presencia de rocas y otras materialidades que en conjunto evidencian una clara interacción entre grupos humanos que incorporaron estos ambientes a sus patrones de movilidad y uso del espacio desde inicios del Holoceno. El registro en Antofagasta de la Sierra de materias primas vegetales para la confección de sistemas de armas para la caza procedentes de Valles y Yungas desde ca.9800 años AP, revela una temprana dependencia de los grupos cazadores-recolectores de la Puna en cuanto a recursos críticos de origen alóctono. Como contraparte, la reciente identificación de obsidianas de origen puneño en la Quebrada de Los Corrales desde ca.7420 años AP, nos permite confirmar un temprano flujo bidireccional Este-Oeste de gente y objetos entre la Puna sur y los Valles orientales, lo cual abre un interesante espectro social y tecnológico a explorar en términos de movilidad e interacción que explique la trayectoria de dichas evidencias.
\end{abstract}

Palabras clave: Puna; Valles; Holoceno medio; interacción; obsidiana.

\begin{abstract}
The comparative study of archaeological evidences belonging to two differentiated geographical areas within northwestern Argentina is addressed in this paper: Antofagasta de la Sierra in the southern Puna (Catamarca) and Quebrada de Los Corrales located in a sector of intermontane valleys (northwest of Tucumán). Both areas have long-term occupational sequences within the Holocene, where we have been able to identify the presence of rocks and other materialities that together show a clear interaction among human groups that incorporated these environments into their patterns of mobility and use of space since the beginning of the Holocene. The record in Antofagasta de la Sierra of vegetal raw materials for the preparation of weapon systems for hunting from Valleys and Yungas from ca.9800 years BP, denotes an early dependence of the hunter-gatherers groups of the Puna on critical resources of allochthonous origin. As a counterpart, the recent identification of obsidians of Puna origin in the Quebrada de Los Corrales from ca.7420 years BP, allows us to confirm an early bidirectional East-West flow of people and objects between the southern Puna and the Eastern Valleys, which opens a interesting social and technological spectrum to be explored in terms of mobility and interaction that explains the trajectory of said evidences.
\end{abstract}

Keywords: Puna; Valleys; Middle Holocene; interaction; obsidian.

\section{Introducción. Problemas y objetivos}

Numerosas investigaciones desarrolladas en el noroeste de Argentina (NOA) permiten afirmar que las primeras ocupaciones humanas se remontan a casi 11000 años atrás, y que las mismas fueron registradas sólo en el 
ámbito de la Puna. Persiste por lo tanto un antiguo problema que alude a una marcada escasez de evidencias arqueológicas de grupos cazadores-recolectores tempranos fuera de la ecorregión puneña, si bien esta representa sólo una tercera parte de la superficie total de esta macrorregión (Martínez 2018).

La mayoría de los sitios arqueológicos en sectores de pre-Puna y Valles intermontanos no superan los 3000 años de antigüedad. No obstante, consideramos que este esquema de "concentración" de sitios del Holoceno temprano y medio en el piso puñeno, es aparente y responde más a la escasez de investigaciones dirigidas específicamente a detectar ocupaciones tempranas en sectores valliserranos, y no necesariamente a factores de preservación diferencial o una dinámica particular del poblamiento temprano del NOA.

Al respecto, nos enfocaremos en un sector de Valles, específicamente en la Quebrada de Los Corrales (Tucumán), donde fue detectado en 2009 el sitio Taller Puesto Viejo 1 (TPV1; 3154 msnm) el cual cuenta con una extensa secuencia crono-estratigráfica que comienza en el Holoceno medio inicial hacia ca. 7820 AP y se extiende hasta ca.1750 AP (Martínez et al. 2013, Oliszewski et al. 2018). Las investigaciones sistemáticas en esta área fueron iniciadas por nuestro equipo en 2005, y hasta la detección de TPV1, el principal corpus de información correspondía a una intensa ocupación agro-pastoril del primer milenio AD (ca.1850-1550 AP). Este componente "formativo" cuenta con numerosos y diferentes tipos de estructuras arquitectónicas de gran obstrusividad en el paisaje, entre las que se destaca la aldea Puesto Viejo con casi un centenar de viviendas tipo "patrón Tafi", un extenso sistema agrícola con andenes (a secano) y numerosos corrales (Oliszewski et al. 2015; Oliszewski 2017).

Dentro de una enorme asimetría difícil de revertir en cuanto al volumen de conocimiento sobre sociedades cazadoras-recolectoras de Puna vs. Valles para el Holoceno temprano y medio, el hallazgo de este tipo de sitios tempranos como TPV1, creemos constituye un valioso aporte que ayudará a mejorar nuestro entendimiento integral sobre los antiguos habitantes del NOA prehispánico. Esto confiere a la Quebrada de Los Corrales (QDLC) una alta relevancia arqueológica, dado que nos ha permitido empezar a evaluar diversos aspectos sobre la movilidad y probable interacción social entre grupos que habitaron sincrónicamente estas dos ecorregiones diferenciadas. Conocer la distribución espacial de recursos líticos -y de otros tipos- dentro de un área o región es crucial para abordar y definir los patrones de movilidad de los grupos humanos del pasado, el acceso de los mismos a diversos espacios, las actividades de intercambio mantenidas con otros y las decisiones asociadas a la tecnología (Bamforth 1986, Nelson 1991, Andrefsky 1994). En definitiva enfocamos a la tecnología como un conjunto de comportamientos que contribuyen a la adaptación humana y a los conjuntos líticos como proveedores de información sobre movilidad, estrategias sociales, subsistencia, riesgo y otros aspectos del comportamiento humano (Carr 1994).

Desde una perspectiva comparativa, evaluamos información previa y reciente de las dos áreas mencionadas: Antofagasta de la Sierra (ANS, Catamarca) en la Puna meridional argentina y QDLC situada en un sector de Valles intermontanos, en el noroeste de Tucumán (Figura 1). En ambas fueron registradas secuencias ocupacionales de larga duración a lo largo del Holoceno, habiéndose identificado además de rocas aptas para la talla, otras materias primas de origen orgánico que apuntarían a la interacción entre grupos cazadores-recolectores de estas áreas desde inicios del Holoceno (Martínez 2003; Martínez et al. 2013). La presentación, análisis e interpretación de estos conjuntos de elementos conforman los objetivos principales de este trabajo, teniendo como eje central el avance en la identificación de los rangos de movilidad e interacción

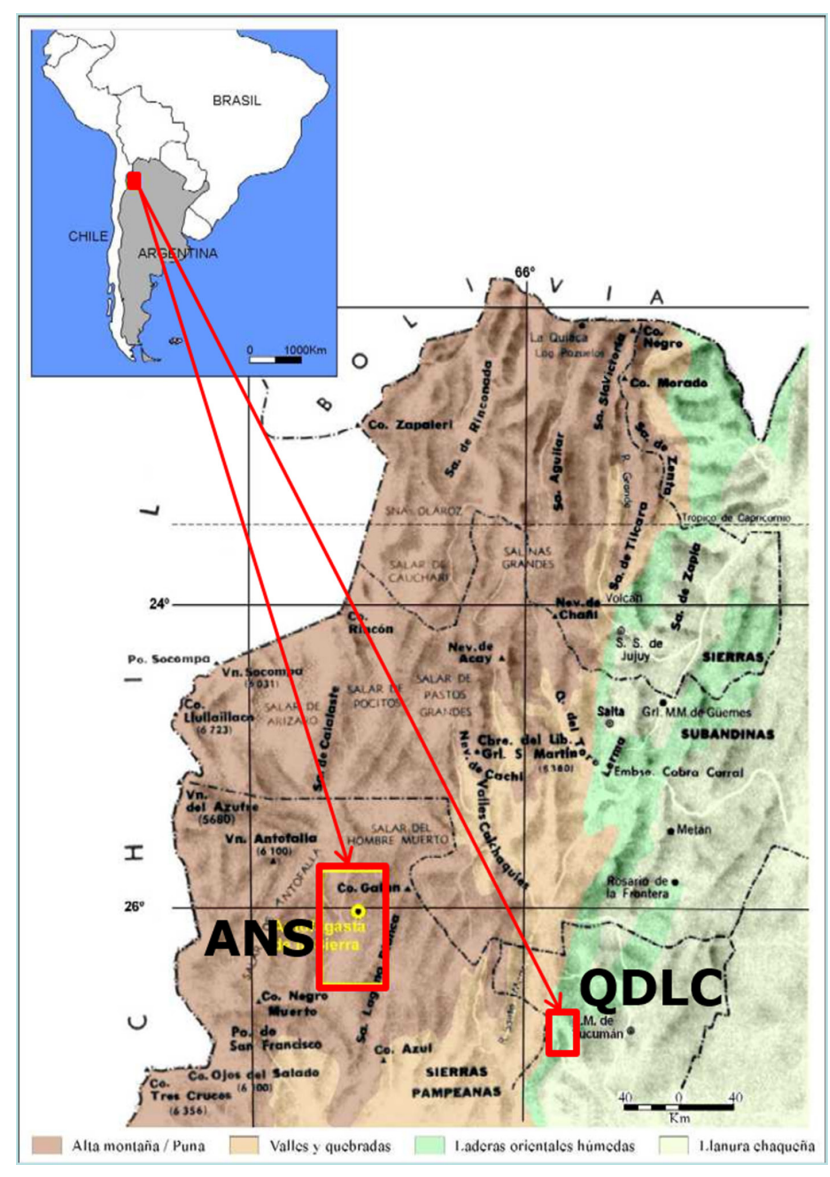

Figura 1. Ubicación geográfica relativa de las áreas de estudio en el NOA: Antofagasta de la Sierra (ANS) y Quebrada de LoS Corrales (QDLC).

Figure 1. Relative geographic location of the study areas in the NWA: Antofagasta de la Sierra (ANS) and Quebrada de Los Corrales (QDLC). 
social entre estas dos áreas durante el Holoceno medio.

\section{Interacción entre Puna sur y Valles del NOA. Evidencias y movilidad.}

El estudio de las fuentes de aprovisionamiento de rocas y de cualquier tipo de materia prima provee información crítica en función de su rol dentro de cualquier sistema social en un determinado espacio y tiempo. En el caso de las materias primas líticas, la localización geográfica precisa de las fuentes es determinante en el estudio de la tecnología lítica y la movilidad resultante para grupos humanos del pasado, lo cual permite dimensionar las estrategias de manejo entre recursos locales y alóctonos. Este mismo planteo aplica también para recursos de origen orgánico.

La comparabilidad entre estas áreas se apoya en parte en la hipótesis de la existencia de tempranas redes de interacción entre grupos humanos de Puna y Valles del NOA desde inicios del Holoceno (Martínez et al. 2013). Este planteo surge en parte por los numerosos recursos de origen alóctono registrados en sitios de Antofagasta de la Sierra desde el Holoceno temprano (desde ca. 9800 AP), que entre otros, se destacan las cañas de Chusquea lorentziana y maderas de Salix humboldtiana para la confección de astiles e intermediarios de sistemas de armas para la caza, las cuales provienen de Valles mesotermales o de bosques de Yungas (Aschero y Martínez 2001, Rodríguez y Martínez 2001, Martínez 2003). Si bien la caza fue la principal actividad de subsistencia, debe remarcarse que la Puna carece -y careció siempre- de especies vegetales aptas para la confección de astiles para los proyectiles de propulsor y/o lanzas, aún considerando las variaciones paleoclimáticas que hubo durante el Holoceno (Martínez 2003, 2007). Fuera de la esfera de lo utilitario, algunas tecno-facturas hechas también con materias primas vegetales de tierras bajas, fueron recuperadas en contextos funerarios del sitio Peñas de las Trampas 1.1 en ANS, asociadas a dataciones de ca. 8400-8000 AP (Martínez 2012, 2014). Se trata de elementos culturales que acompañan a los restos óseos humanos, tales como cordeles y fragmentos de malla de color rojizo hechos con Acrocomia chunta ("palmera chonta") la cual proviene de un sector de Yungas (Rodríguez y Aschero 2005). A esto se suman numerosas cuentas de collar hechas con endocarpos de Condalia buxifolia ("piquillín grande"; M. L. Pochettino com. pers.) procedentes de selvas de Yungas también, e incluso del Chaco Seco que estaría más alejado aún del piso puneño. Todos estos elementos foráneos detectados en contextos puneños tempranos, marcan un claro vector de movilidad orientado hacia pisos altitudinales orientales más bajos.

Geográficamente, debe destacarse que QDLC forma parte del espacio actualmente conocido como Abra de El Infiernillo, un área de "paso" natural y obligado desde la Puna sur para poder acceder en sentido Oeste-Este a la ecorregión de las Yungas y sus recursos. En relación a la tecnología lítica de QDLC, la información de la secuencia estratigráfica del sitio TPV1 sintetiza el panorama general de las estrategias de aprovisionamiento y uso de los diferentes tipos de rocas aptas para la talla para el lapso ca.7820-1750 AP en dicha quebrada. El análisis de desechos de talla procedentes de una trinchera de 3 $\mathrm{m}^{2}$ excavada en el Sector I de TPV1 (Cuadrículas A1, B1 y C1; definido como un campamento base de actividades múltiples) denota un dominante uso de materias primas locales, alcanzando el $93 \%$ en toda la secuencia,

Tabla 1. Fuentes de procedencia identificadas y concentraciones de elementos traza en partes por millón de las lascas de obsidiana analizadas por Fluorescencia de rayos X del sitio TPV1.

Table 1. Identified sources of origin and trace-element concentrations in parts per million of the obsidian flakes analyzed by $X$-ray Fluorescence of the TPV1 site.

\begin{tabular}{|c|c|c|c|c|c|c|c|c|c|c|c|}
\hline $\begin{array}{c}\text { Muestra } \\
\text { (N=9) }\end{array}$ & Nivel & Fuente & Mn & Fe & Zn & Rb & Sr & Y & Zr & Nb & Th \\
\hline TPV008 & 1 & $\begin{array}{c}\text { Laguna } \\
\text { Cavi }\end{array}$ & 708,4 & 5742,8 & 75,5 & 516,2 & 0,0 & 36,6 & 62,4 & 31,3 & 25,1 \\
\hline TPV001 & 3 & Archibarca & 502,0 & 11923,4 & 103,9 & 114,8 & 296,0 & 12,7 & 105,7 & 15,8 & 17,9 \\
\hline TPV009 & 5 & $\begin{array}{c}\text { Laguna } \\
\text { Cavi }\end{array}$ & 931,9 & 6179,2 & 219,4 & 421,4 & 1,9 & 27,1 & 32,9 & 22,8 & 26,4 \\
\hline TPV005 & 7 & $\begin{array}{c}\text { Cueros de } \\
\text { Purulla- } \\
\text { Chascón }\end{array}$ & 944,5 & 12412,5 & 109,3 & 164,7 & 277,8 & 19,1 & 122,7 & 24,4 & 17,8 \\
\hline TPV003 & 12 & $\begin{array}{c}\text { Ona-Las } \\
\text { Cuevas }\end{array}$ & 417,6 & 8792,0 & 89,7 & 215,4 & 128,7 & 6,3 & 71,7 & 13,1 & 20,2 \\
\hline TPV006 & 12 & $\begin{array}{c}\text { Laguna } \\
\text { Cavi }\end{array}$ & 723,3 & 6081,8 & 97,4 & 525,0 & 0,0 & 30,6 & 38,7 & 32,0 & 27,4 \\
\hline TPV004 & 15 & $\begin{array}{c}\text { Laguna } \\
\text { Cavi }\end{array}$ & 997,4 & 6744,1 & 142,7 & 568,3 & 0,0 & 30,6 & 47,6 & 27,5 & 34,8 \\
\hline TPV007 & 15 & $\begin{array}{c}\text { Laguna } \\
\text { Cavi }\end{array}$ & 859,4 & 5881,6 & 105,3 & 552,5 & 0,0 & 34,9 & 47,4 & 34,1 & 32,9 \\
\hline TPV011 & 15 & $\begin{array}{c}\text { Laguna } \\
\text { Cavi }\end{array}$ & 712,0 & 6297,1 & 130,2 & 502,1 & 0,0 & 36,5 & 42,3 & 26,3 & 30,1 \\
\hline
\end{tabular}


dado por andesita y cuarzo (Martínez et al. 2013). Sin embargo, se registró una covariación inversa de uso a lo largo de toda la secuencia entre estas dos rocas. Desde los niveles inferiores (Niveles 17-18, ca.7820 AP), la frecuencia de rocas andesíticas sobrepasa notablemente a la del cuarzo. Considerando estas dos materias primas, la dominancia de andesita comienza a disminuir gradualmente desde el Nivel 10 (ca.6490 AP) y en el Nivel 6 (ca.3490 AP) esta proporción cambia abruptamente volviéndose más frecuente el cuarzo, tendencia que se mantiene hacia momentos posteriores.

Dentro de las rocas de uso minoritario, se destaca el registro de desechos de talla de obsidiana a lo largo de la secuencia estratigráfica desde las ocupaciones más antiguas de TPV1 (ca.7420 AP; Nivel 15), hasta el 1er milenio de la Era Cristiana (ca.1750 AP; Nivel 3). La muestra total se compone sólo de nueve desechos de talla, todas lascas internas de tamaño microlasca según el análisis técnico-morfológico realizado (sensu Aschero 1975, 1983), lo cual alude en todos los casos a la regularización final y/o mantenimiento de artefactos mediante retoque y microrretoque a presión. Si bien la cantidad de lascas de obsidiana es muy reducida, denota una alta relevancia arqueológica por el potencial

Tabla 2. Diferentes tipos de obsidianas identificados por cuadrículas y niveles en el sitio TPV1 (NMD = número mínimo de desechos de talla)

Table 2. Different types of obsidians identified by grids and levels in TPV1 site (MND = minimum number of debris)

\begin{tabular}{|c|c|c|c|c|c|}
\hline Nivel & $\mathrm{N}$ & Cuadrícula A1 & Cuadrícula B1 & Cuadrícula C1 & Cronología \\
\hline 1 & 1 & & & $\begin{array}{l}\text { Laguna Cavi } \\
\text { (TPV008) }\end{array}$ & \\
\hline 2 & - & & & & \\
\hline 3 & 1 & $\begin{array}{l}\text { Archibarca } \\
\text { (TPV001) }\end{array}$ & & & $1750 \pm 20 \mathrm{AP}$ \\
\hline 4 & - & & & & \\
\hline 5 & 1 & & & $\begin{array}{l}\text { Laguna Cavi } \\
\text { (TPV009) }\end{array}$ & $3490 \pm 25 \mathrm{AP}$ \\
\hline 6 & - & & & & \\
\hline 7 & 1 & & $\begin{array}{l}\text { Cueros de Purulla- } \\
\text { Chascón (TPV005) }\end{array}$ & & \\
\hline 8 & - & & & & \\
\hline 9 & - & & & & \\
\hline 10 & - & & & & \\
\hline 11 & - & & & & \\
\hline 12 & 2 & $\begin{array}{c}\text { Ona-Las Cuevas } \\
\text { (TPV003) }\end{array}$ & $\begin{array}{l}\text { Laguna Cavi } \\
\text { (TPV006) }\end{array}$ & & $6490 \pm 30 \mathrm{AP}$ \\
\hline 13 & - & & & & \\
\hline 14 & - & & & & \\
\hline 15 & 3 & $\begin{array}{l}\text { Laguna Cavi } \\
\text { (TPV004) }\end{array}$ & $\begin{array}{l}\text { Laguna Cavi } \\
\text { (TPV007) }\end{array}$ & $\begin{array}{l}\text { Laguna Cavi } \\
\text { (TPV011) }\end{array}$ & $7420 \pm 25 \mathrm{AP}$ \\
\hline 16 & - & & & & \\
\hline 17 & - & & & & \\
\hline 18 & - & & & & $7820 \pm 30 \mathrm{AP}$ \\
\hline & & $\mathrm{N}=3(0,42 \%)$ & $\mathrm{N}=3(0,31 \%)$ & $N=3(0,36 \%)$ & \\
\hline & & NMD = 701 & $\mathrm{NMD}=939$ & $\mathrm{NMD}=828$ & \\
\hline
\end{tabular}

arqueométrico que tiene su firma química para poder definir su procedencia geográfica con bastante precisión. Todos los desechos de obsidiana recuperados en los distintos niveles de la secuencia de TPV1 $(\mathrm{N}=9)$, fueron analizados mediante la técnica de Fluorescencia de rayos X (FRX) en el laboratorio MURR (Missouri, EEUU; dirigido por M. Glascock). En base a este análisis se identificó el uso de cuatro fuentes distintas en toda la secuencia que va desde ca. 7420-1750 AP (ver Tabla 1).

Estas cuatro fuentes se ubican todas en el ámbito de la Puna meridional argentina: Laguna Cavi, OnaLas Cuevas, Cueros de Purulla-Chascón y Archibarca, cuya distancia mínima es de ca.140 km lineales desde QDLC. En las capas inferiores de TPV1 (Niveles 15 a 12; ca.7420-6490 AP) pudo determinarse la presencia de obsidianas procedentes de dos canteras: Laguna Cavi y Ona-Las Cuevas. Posteriormente, se suma la cantera de Cueros de Purulla-Chascón hacia ca.3490 AP (Nivel 7), y ya en momentos "formativos" (Nivel 3; ca.1750 AP) surge el uso de la cantera de Archibarca, la más alejada de todas las fuentes desde QDLC (ca.310 km lineales) (ver Tabla 2).

La bajísima frecuencia relativa en la que se encuentran representadas las lascas de obsidiana frente a las otras materias primas locales, nueve especímenes frente a un total $\mathrm{NMD}=$ 2468, deja claro que no es posible obtener ningún tipo de tendencia en cuanto a variaciones en el uso de distintas fuentes identificadas a lo largo de la secuencia. No obstante, destacamos lo significativo de este bajo número de lascas de obsidiana, ya que en los distintos niveles estratigráficos de TPV1, se encuentran representadas cuatro de las cinco fuentes existentes en la Puna meridional argentina. La única fuente sin registro en TPV1 es la del Salar del Hombre Muerto. Este panorama tenderá a mejorar ya que contamos con un mayor número de lascas y artefactos de esta materia prima (provienen de una reciente excavación de un sector contiguo), pero su análisis aún está en curso.

Para la microrregión ANS, sintéticamente diremos que fue detectada una gran cantidad de fuentes de materias primas líticas que fueron utilizadas con variable intensidad desde las primeras ocupaciones registradas en el área hacia ca.10.000 años AP (Aschero et al. 2002-2004). En este momento se inicia una larga y variable trayectoria en las estrategias de aprovisionamiento y uso de los recursos líticos, empleados para 
Figura 2. Ubicación geográfica de las canteras de obsidiana (triángulos) de la Puna sur argentina en relación a la posición de los sitios de ANS y QDLC para el lapso ca.9000$3500 \mathrm{AP}$

Figure 2. Geographic location of the obsidian quarries (triangles) of the southern Argentine

Puna in relation to the position of the ANS and QDLC sites for ca.9000$3500 B P$.

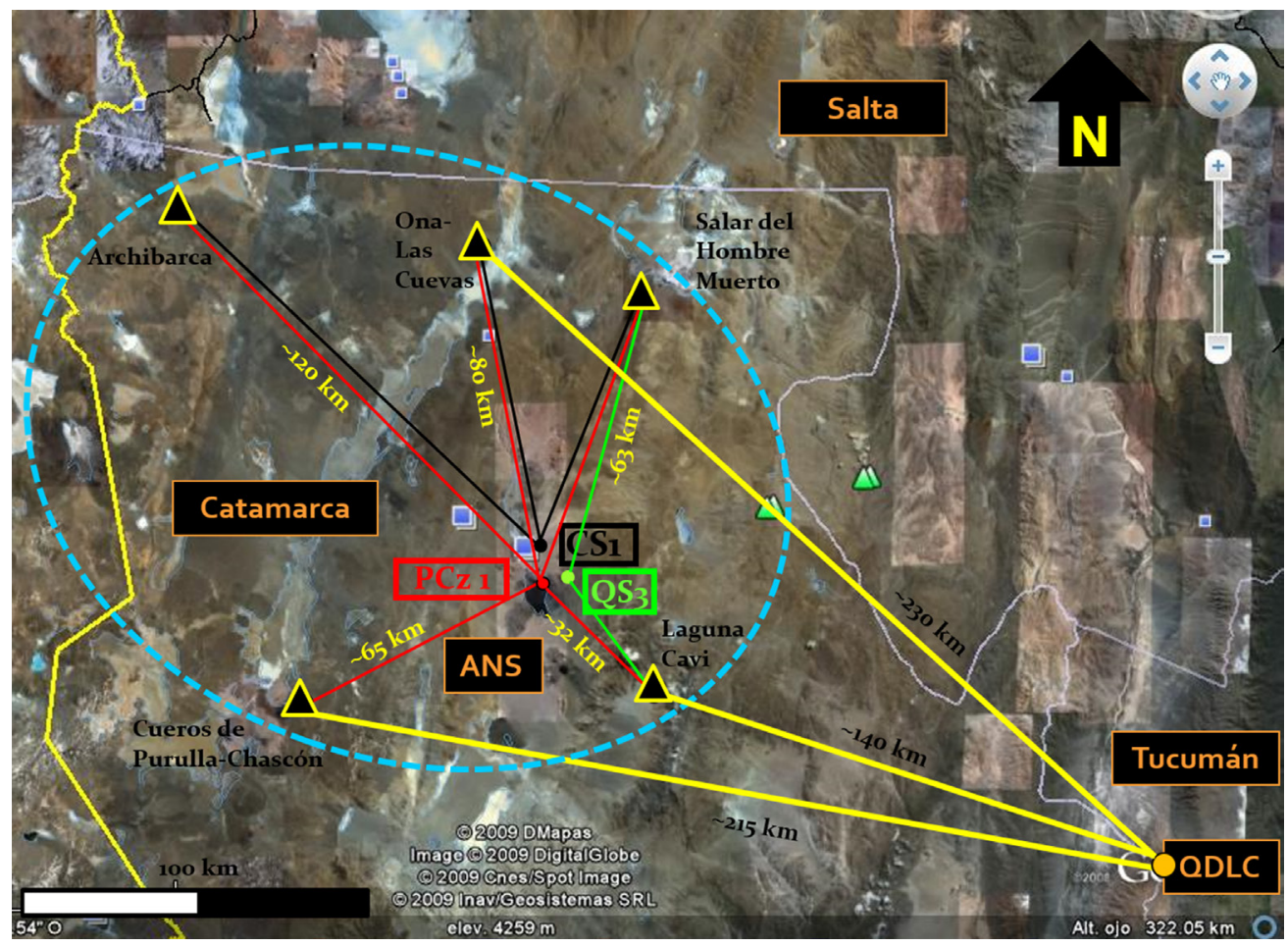

la confección de una amplia variedad de artefactos (Aschero et al. 1991; Aschero et al. 1993-94; Escola 2000; Hocsman 2006; Manzi 1999; Martínez 2003; Pintar 1996). De acuerdo a esta información disponible para ANS durante el Holoceno temprano y medio, poco más de $82 \%$ de las canteras explotadas son locales (14 de 17) y se ubican dentro de un radio de $15 \mathrm{~km}$ desde un centro equidistante del área de estudio (río Las Pitas), destacándose que dentro de este mismo radio se ubican más del $80 \%$ de los sitios arqueológicos relevados en esta microrregión. Se destaca que de los 16 tipos de roca utilizadas, 13 de ellas $(81,25 \%)$ corresponden a distintas variantes de rocas de tipo basálticas de muy buena calidad, lo cual convierte a ANS en un territorio con gran abundancia y diversidad de recursos líticos. En cuanto al uso y circulación de obsidianas durante el lapso ca.9000-6000 AP, recientes estudios de Fluorescencia de Rayos X sobre materiales de los sitios Quebrada Seca 3 (QS3), Cueva Salamanca 1 (CS1) y Peñas de la Cruz 1 (PCz1) permitieron identificar el uso sincrónico -aunque variable- de las cinco canteras: Laguna Cavi, Ona-Las Cuevas, Cueros de Purulla-Chascón, Salar del Hombre Muerto y Archibarca (Pintar et al. 2016). En la Figura 2 puede observarse la situación geográfica relativa de estas canteras en relación a la ubicación y distancias lineales de los sitios de ANS y QDLC.

Otra línea de evidencia que apoya nuestro planteo sobre las interacciones entre ambas áreas, se relaciona con la presencia en ANS y QDLC de puntas de proyectil líticas del mismo tipo morfológico (Martínez et al. 2016). Se trata de dos diseños compartidos correspondientes al Holoceno temprano y medio inicial. Uno de estos, refiere a puntas apedunculadas de limbo triangular asignadas en ANS al tipo morfológico Quebrada Seca A (QSA) asociadas al uso de propulsor (Martínez 2003, 2007). Este tipo de puntas se asocia a una amplia dispersión dentro de la Puna argentina y norte de Chile, cronológicamente asignadas a la transición Pleistoceno-Holoceno. El otro diseño detectado en ambas áreas de estudio, se homologa al tipo morfológico definido para ANS como Peñas de la Cruz A (PCZA), tratándose de puntas apedunculadas de limbo lanceolado, vinculadas también al propulsor como sistema de arma durante el Holoceno medio inicial hacia ca. 7900-7000 años AP (Martínez et al. 2016).

\section{Discusión y consideraciones finales}

Las evidencias presentadas confirman con mayor fuerza la existencia de tempranos mecanismos sociales de interacción entre grupos cazadores-recolectores, cuyos rangos de movilidad cubrieron las áreas entre Puna surANS y Valles-QDLC, al menos desde el Holoceno medio inicial en adelante. Obsidianas puneñas en Valles desde ca.7420 AP, y cañas y maderas para la fabricación de astiles de las Yungas/Valles en la Puna desde ca.9800 $A P$, evidencian sin embargo un esquema de interacción muy particular, ya que los grupos puneños dependieron obligadamente de los recursos vegetales mencionados, para poder confeccionar los sistemas de armas para la caza de camélidos. A la inversa, los grupos humanos de Valles -como los habitantes de QDLCfueron autosuficientes en este sentido y no tuvieron ningún tipo de dependencia de recursos alóctonos para su subsistencia. Como fuera mencionado, la alta disponibilidad local de rocas de buena calidad (andesita y cuarzo principalmente), sumado a la proximidad de áreas con varas de Salix y cañas de Chusquea para astiles e intermediarios, configuraron un esquema de aprovisionamiento de bajo costo logístico para los ocupantes de QDLC. 
Si bien los elementos alóctonos registrados en ANS desde el Holoceno temprano permitían proponer un vínculo con territorios orientales pre-puneños, esta conexión era unidireccional, de arriba hacia abajo. El hallazgo en QDLC de obsidianas procedentes de canteras de Puna sur-ANS, confirma por primera vez para el Holoceno medio, la existencia de algún tipo de interacción bidireccional y/o intercambio de elementos foráneos entre grupos humanos que ocuparon ambas áreas o nodos (sensu Nielsen 2006). La distancia desde QDLC hasta las canteras puneñas de obsidianas varía entre 140 y 310 km lineales hacia el oeste. En este sentido, cobra mayor relevancia aún el registro de obsidianas en Valles-QDLC, ya que se registran desde el inicio de las ocupaciones cazadoras-recolectoras hacia ca.7420 años AP, lo cual permite avanzar en la comprensión del sistema de interacción que subyace y motoriza estos movimientos de gente y objetos durante milenios entre estas dos ecorregiones diferenciadas.

En cuanto a la explotación de las diferentes canteras de obsidianas en ANS, los resultados muestran una fuerte tendencia hacia la existencia de una circunscripción territorial en la Puna meridional argentina, ya que el aprovisionamiento de obsidianas se restringe geográficamente a canteras dentro de dicha región exclusivamente (Pintar et al. 2016). Más enfáticamente diremos que todas las canteras identificadas y explotadas para este lapso, se ubican en el interior del departamento Antofagasta de la Sierra de la actual provincia de Catamarca. Considerando un punto central promediado para los tres sitios tratados, la fuente más lejana es Archibarca a ca.120 km (Noroeste), siendo esta distancia el rango máximo de movilidad -dentro de la Puna- para los grupos que habitaron ANS. Prueba de esto, es la no utilización durante ca.9000-6000 AP (y hasta ca.3500 AP) de las canteras de Quirón en la Puna de Salta a ca.170 km al norte, y Valle Ancho a ca. $200 \mathrm{~km}$ al suroeste en la Puna de Catamarca (Pintar et al. 2016). Esto pone sobrerrelieve la presencia de obsidianas puneñas en Valles-QDLC desde el inicio de las ocupaciones de TPV1, ubicado a ca.165 km hacia el Este de ANS. Por lo tanto existe un vector muy claro de interacción humana en sentido Este-Oeste entre grupos cazadores-recolectores de Puna sur-ANS y Valles-QDLC para este mismo momento, pero no en sentido NorteSur dentro del ámbito de la Puna propiamente. A pesar de esta circunscripción en el piso puneño, de algún modo el sistema de producción lítica (sensu Ericson 1984) de las obsidianas -unificando todas las canteras puneñas- amplió su alcance territorial hasta los VallesQDLC y no hacia la Puna intermedia y Puna norte para el Holoceno temprano y medio. Consideramos que esta situación es una consecuencia directa de una estrategia demovilidad generada principalmente por la búsqueda, aprovisionamiento y/o eventual intercambio de cañas y maderas como recursos críticos para los cazadores de Puna sur-ANS.
Si bien este trabajo intenta ser un aporte en la discusión sobre la movilidad en sentido amplio, sabemos de la alta complejidad y variabilidad que existe en tiempo y espacio dentro de los diversos casos de grupos cazadores-recolectores presentes y pasados (Binford 1980, Kelly 1992). La detección de sitios tempranos fuera de la Puna como el caso de TPV1-QDLC, es un primer paso que enriquecerá el debate y permitirá explicar las distintas dimensiones sociales, tecnológicas y ambientales que configuraron el modo de vida de cientos de generaciones durante parte del Holoceno. No obstante consideramos que aún son muchos los elementos ausentes y/o aspectos pendientes de resolver para poder establecer modelos hipotéticos válidos de movilidad entre los nodos Puna-Valles. Por ejemplo la información paleoambiental disponible para Puna es por lejos mucho más precisa en relación a las ecorregiones de pre-Puna y Valles. A lo largo del Holoceno, principalmente en la transición del temprano al medio (ca.8200 AP), hubo drásticos cambios ambientales registrados en el ámbito puneño, caracterizados por condiciones de creciente aridez (Tchilinguirian y Morales 2013), que con seguridad incidieron en las estrategias de movilidad a nivel de meso y macro-escala (sensu Dincauze 2000). Este esquema de un Holoceno medio hiperárido aún no puede extrapolarse con confianza a las ecorregiones de menor altitud al Este de la Puna.

Por otra parte, aunque no contamos con un correlato material, muy probablemente hubo otro tipo de factores que se combinaron para impulsar la movilidad de los grupos humanos de ambos nodos dentro de este esquema arriba-abajo/abajo-arriba. Nos referimos por ejemplo al funcionamiento de redes de parentesco entre grupos establecidos en diferentes sectores internodales (sensu Nielsen 2006) que aún no fueron detectados.

En conjunto nuestra interpretación se aproxima al modelo propuesto inicialmente por Rick (1980) denominado "Puna-Plus", al cual adhiere Pintar (2008) para los grupos humanos que ocuparon la Puna meridional argentina durante el Holoceno temprano y medio. Este modelo alude a la permanencia de grupos cazadores-recolectores en el piso puneño, pero que practicaban el intercambio de recursos con gente que ocupaban otras zonas ecológicas. De todos modos consideramos que hay algunos aspectos que deben mejorarse, ya que notamos que el planteo de Pintar (2008) tiene implícito un excesivo peso puesto en pensar unidireccionalmente todo desde la Puna hacia los Valles orientales. Por otra parte, debe tenerse en cuenta que el espacio entre ANS y QDLC representa un factor extra de complejidad para la circulación, dada la accidentada orografía natural de este territorio intermedio con altos contrastes altitudinales y ecológicos, los cuales pudieron operar como barreras geográficas. En este sentido, debemos avanzar hacia un mejor dimensionamiento y/o jerarquización de estos espacios en términos biogeográficos (sensu Borrero 2004) a fin de lograr una 
mejor aproximación a la dinámica de las interacciones bajo estudio.

Como fuera mencionado, es la Puna y su gente la que depende de recursos críticos procedentes de ecorregiones distantes del Este y no a la inversa. La autosuficiencia de recursos en Valles-QDLC para la confección de sistemas de armas, marca una fuerte diferencia que debe ser tenida muy en cuenta, por ejemplo en los casos de los dos tipos morfológicos de puntas de proyectil idénticos en ambas áreas (QSA y PCzA). Estas "conexiones" tecnológicas de diseño incluso permiten proponer que fue en el ámbito de los Valles-QDLC donde se resolvieron inicialmente las técnicas o modos de enmangue de estas puntas de proyectil, dada la fácil accesibilidad a cañas y maderas en esta ecozona. Las puntas adscriptas al tipo Peñas de la Cruz A (rango 8900-7000 AP) halladas en QDLC fueron confeccionadas sobre andesitas locales, y abren la probabilidad de que este diseño se origine en esta área y luego sea replicado en ANS con materias primas puneñas (vulcanitas).

Este trabajo intenta aportar nuevas evidencias y propuestas a fin de dar cuenta de los nuevos interrogantes que surgen a distintas escalas de análisis, y creemos deben ser abordados mediante análisis multivariados que contemplen la combinación y complementariedad de diferentes líneas de evidencia, para lograr una comprensión verdaderamente integral de los esquemas de movilidad implementados por parte de los grupos humanos que ocuparon estas dos microrregiones del NOA durante el Holoceno medio.

Yerba Buena (Tucumán), 30 de agosto de 2019.-

\section{Agradecimientos}

En primer lugar queremos agradecer a Fabiana Skarbun, Manuel Cueto y Ariel Frank quienes coordinaron en septiembre de 2018 el Simposio "Desde las fuentes: Avances en los estudios de selección y aprovisionamiento de rocas y sus implicancias en las prácticas", donde fue presentado este trabajo en el marco del $1^{\circ}$ CAELA (Córdoba). Las fuentes de financiamiento que permitieron el desarrollo de estas investigaciones son el PICT 20131700 y el PIP-CONICET 0222, ambos dirigidos por el primer autor. Queremos agradecer también a Elizabeth Pintar por su valiosa ayuda para poder concretar los análisis de FRX en Missouri. Finalmente a los dos evaluadores anónimos, cuyos comentarios contribuyeron a mejorar nuestro trabajo.

\section{Referencias citadas}

Andrefsky, W. (1994). Raw material availability and the organization of technology. American Antiquity 59 (1): 21-34.

Aschero, C. A. 1975. Ensayo para una clasificación morfológica de artefactos líticos aplicada a estudios tipológicos comparativos. Informe presentado al Consejo Nacional de Investigaciones Científicas y Técnicas (CONICET). Buenos Aires, Argentina. Ms.

Aschero, C. A. 1983. Ensayo para una clasificación morfológica de artefactos líticos. Apéndice A y B. Manuscrito inédito. Universidad de Buenos Aires. Buenos Aires, Argentina. Ms.

Aschero, C. A., D. Elkin y E. L. Pintar. (1991). Aprovechamiento de recursos faunísticos y producción lítica en el precerámico tardío. Un caso de estudio: Quebrada Seca 3 (Puna Meridional Argentina). H. Niemeyer (ed.), Actas del XI Congreso Nacional de Arqueología Chilena, pp. 101-114. Tomo II. Imprenta Caballero, Santiago de Chile.

Aschero, C. A., L. Manzi y A. G. Gómez. (1993-94). Producción lítica y uso del espacio en el nivel 2b4 de Quebrada Seca 3. Relaciones 19: 191-214.

Aschero, C. A., J. G. Martínez. (2001). Técnicas de Caza en Antofagasta de la Sierra, Puna Meridional Argentina. Relaciones 26: 215-241.

Aschero, C. A., P. S. Escola, S. Hocsman y J. G. Martínez. (20022004). Recursos líticos en escala microrregional Antofagasta de la Sierra, 1983-2001. Arqueología 12: 9-36.

Bamforth, D. (1986). Technological efficiency and tool curation. American Antiquity 51 (1): 38-50.

Binford, L. R. (1980). Willow smoke and dogs' tails: HunterGatherer settlement systems and archaeological site formation. American Antiquity 45(1): 4-20.

Borrero, L. A. (2004). The Archaeozoology of Andean "Dead Ends" in Patagonia: Living near the Continental Ice Cap. En Mondini, M.S., A.S. Muñoz y S. Wickler (eds.), Colonisation, Migration and Marginal Areas. A Zooarchaeological approach, pp. 55-61. Oakville, Oxbow Books.

Carr, P. (1994). The organization of technology: impact and potencial. P. Carr (ed.), The Organization of North American Prehistoric Chipped Stone Tool Technologies, pp. 1-8. Archaeological Series 7. International Monographs in Prehistory. Ann Arbor, Michigan.

Dincauze, D. (2000). Environmental Archaeology. Principles and Practice. Cambridge, Cambridge University Press.

Ericson, J. E. (1984). Toward the analysis of lithic production systems. En: J. E. Ericson y B. A. Purdy (eds.), Prehistoric Quarries and Lithic Production, Cambridge University Press, UK.

Escola, P. S. (2000). "Tecnología lítica y contextos agropastoriles tempranos". Facultad de Filosofía y Letras, Universidad de Buenos Aires, Argentina. Tesis Doctoral inédita.

Hocsman, S. (2006). "Producción Lítica, Variabilidad y Cambio en Antofagasta de la Sierra -ca. 5500-2000 AP-". Facultad 
de Ciencias Naturales, Universidad Nacional de La Plata, Argentina. Tesis Doctoral inédita.

Kelly, R. (1992). Mobility/sedentism: concepts, archaeological measures and effects. Annual Review of Anthropology 21: 4366.

Manzi, L. M. (1999). “Análisis de la distribución espacial de conjuntos líticos en el sector de quebradas de altura e intermedio de la cuenca del Río Las Pitas. Antofagasta de la Sierra. Catamarca". Facultad de Filosofía y Letras, Universidad de Buenos Aires, Argentina. Tesis Doctoral inédita.

Martínez, J. G. (2003). “Ocupaciones Humanas Tempranas y Tecnología de Caza en la Microrregión de Antofagasta de la Sierra (10000-7000 AP)". Facultad de Ciencias Naturales e Instituto Miguel Lillo. Universidad Nacional de Tucumán, Argentina. Tesis Doctoral inédita.

Martínez J. G. (2007). Ocupaciones humanas tempranas y tecnología de caza en Antofagasta de la Sierra, Puna Meridional Argentina (10000-7000 AP). Cazadores-Recolectores del Cono Sur. Revista de Arqueología 2:129-150.

Martínez J. G. (2012). Evidence of early human burials in the Southern Argentinian Puna. L. Miotti, M. Salemme, N. Flegenheimer, T. Goebel (eds.), Southbound: Late Pleistocene Peopling of Latin America, pp. 75-78, Center for Studies of the First Americans, College Station, Texas.

Martínez, J. G., E. Mauri, C. Mercuri, M. Caria y N. Oliszewski. (2013). Mid-Holocene human occupations inTucumán (Northwest of Argentina). Quaternary International 307:86-95.

Martínez, J. G. (2014). Contributions to the knowledge of natural history and archaeology of hunter-gatherers of Antofagasta de la Sierra (Southern Argentinian Puna): the case of Peñas de las Trampas 1.1. E. L. Pintar (ed.), Huntergatherers from a high-altitude desert. People of the Salt Puna (northwest Argentina), pp. 71-93. BAR International Series, 2641, 7, Archaeopress, Oxford.

Martínez, J. G., J. A. Funes Coronel y Eduardo P. Mauri. (2016). Comparando Peras con Manzanas?...Análisis Comparativo de Puntas de Proyectil y Sistemas de Armas Prehispánicos entre QDLC-ANS. Implicancias para el Estudio de la Movilidad entre Puna y Valles del NOA. A. L. Aquino, M. S. Caro y G. E. Ruiz de Bigliardo (eds.), Actas del XIX Congreso Nacional de Arqueología Argentina, pp. 3077-3079. Universidad Nacional de Tucumán.

Martínez, J. G. (2018). Sociedades prehispánicas de la Puna argentina: desde el poblamiento temprano hasta los inicios de la producción pastoril y agrícola. R. Grau, J. Babot, A. Izquierdo y A. Grau (eds.), La Puna Argentina: Naturaleza y Cultura. Serie de Conservación de la Naturaleza 24: 273-294. Editorial Fundación Miguel Lillo, Tucumán

Nelson, M. (1991). The study of technological organization. Archaeological Method and Theory Vol. 3: 57-100.
Nielsen, A. (2006). Estudios internodales e interacción interregional en los Andes Circumpuneños: Teoría, método y ejemplos de aplicación. H. Lechtman (ed.), Esferas de interacción prehistóricas y fronteras nacionales modernas en los Andes Sur Centrales, pp. 29-62. Lima, Instituto de Estudios Peruanos e Institute of Andean Research.

Oliszewski, N., J. G. Martínez, E. Di Lullo, M. Gramajo Bühler, G. Arreguez, H. Cruz, E. Mauri, C. Mercuri, A. Muntaner y G. Srur. (2015). Contribuciones al estudio de sociedades aldeanas en el Noroeste Argentino: el caso de la quebrada de Los Corrales (El Infiernillo, Tucumán). A. Korstanje, M. Lazzari, M. Basile, F. Bugliani, V. Lema, L. Pereyra Domingorena y M. Quesada (eds.), Crónicas Materiales Precolombinas. Arqueología de los Primeros Poblados del Noroeste Argentino, pp. 51-79. Sociedad Argentina de Antropología, Buenos Aires.

Oliszewski, N. (2017). Las aldeas "Patrón Tafi" del sur de Cumbres Calchaquíes y norte del Sistema del Aconquija. Comechingonia Revista de Arqueología21(1):205-232.

Oliszewski, N., J. G. Martínez, G. A. Arreguez, C. M. Gramajo Bühler y M. E. Naharro. (2018). La transición vista desde los valles intermontanos del noroeste argentino: nuevos datos de la Quebrada de Los Corrales (El Infiernillo, Tucumán, Argentina). Chungara Revista de Antropología Chilena 50(1): 71-86.

Pintar, E. L. (1996). "Prehistoric holocene adaptations to the Salt Puna of Northwestern Argentina". Southern Methodist University, Fayetteville, North Carolina, USA. Tesis Doctoral inédita.

Pintar, E. L. (2008). High Altitude Deserts: Hunter-Gatherers from the Salt Puna, North West, Argentina. International Journal of South American Archaeology 2: 47-55.

Pintar, E. L., J. G. Martínez, C. A. Aschero y M. D. Glascock. (2016). Obsidian use and mobility during the Early and Middle Holocene in the Salt Puna, NW Argentina. Quaternary International422: 93-108.

Rick, J. W. (1980). Prehistoric Hunters of the High Andes. Academic Press, New York.

Rodríguez, M. F., J. G. Martínez. (2001). Especies vegetales alóctonas como recursos arqueológicos en el ámbito puneño. Publicación Especial de la Asociación Paleontológica Argentina 8: 139-145.

Rodríguez M. F., C. A. Aschero. (2005). Acrocomia chunta (Arecaceae) Raw material for cord making in the Argentinean Puna. Journal of Archaeological Science 32: 1532-1542.

Tchilinguirian, P., M. R. Morales. (2013). Mid-holocene Paleoenvironments in Northwestern Argentina: Main Patterns and Discrepancies. Quaternary International 307: 14-23. 